\title{
Regulation of transgene expression in genetic immunization
}

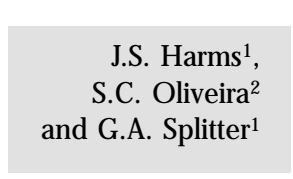

\author{
${ }^{1}$ Department of Animal Health and Biomedical Sciences, University of Wisconsin, \\ Madison, WI, USA \\ 2Departamento de Bioquímica e Imunologia, Instituto de Ciências Biológicas, \\ Universidade Federal de Minas Gerais, Belo Horizonte, MG, Brasil
}

\section{Correspondence \\ J.S. Harms \\ AHABS \\ 1655 Linden Drive \\ Madison, WI 53706 \\ USA \\ Fax: + 1-608-262-7420 \\ E-mail: harms@ahabs.wisc.edu \\ Research supported by the Robert Draper Technology Innovation Fund (No. 135-0546) of the University of Wisconsin, Madison. \\ Presented at the International Symposium "The Third Revolution on Vaccines: DNA Vaccines", Belo Horizonte, MG, Brasil, November 3-7, 1997.}

Received O ctober 5, 1998 Accepted November 3, 1998

\section{Abstract}

The use of mammalian gene expression vectors has become increasingly important for genetic immunization and gene therapy as well as basic research. Essential for the success of these vectors in genetic immunization is the proper choice of a promoter linked to the antigen of interest. Many genetic immunization vectors use promoter elements from pathogenic viruses including SV40 and CMV. Lymphokines produced by the immune response to proteins expressed by these vectors could inhibit further transcription initiation by viral promoters. Our objective was to determine the effect of IFN- $\gamma$ on transgene expression driven by viral SV40 or CMV promoter/enhancer and the mammalian promoter/enhancer for the major histocompatibility complex class I (MHC I) gene. We transfected the luciferase gene driven by these three promoters into 14 cell lines of many tissues and several species. Luciferase assays of transfected cells untreated or treated with IFN- $\gamma$ indicated that although the viral promoters could drive luciferase production in all cell lines tested to higher or lower levels than the MHC I promoter, treatment with IFN- $\gamma$ inhibited transgene expression in most of the cell lines and amplification of the MHC I promoterdriven transgene expression in all cell lines. These data indicate that the SV40 and CMV promoter/enhancers may not be a suitable choice for gene delivery especially for genetic immunization or cancer cytokine gene therapy. The MHC I promoter/enhancer, on the other hand, may be an ideal transgene promoter for applications involving the immune system.

\section{Introduction}

During the past few years, the use of mammalian gene expression vectors for new methods such as genetic immunization has become increasingly important. Recent success with DNA vaccines indicates their enormous future potential in diverse fields such as infectious diseases, allergy and cancer (1$6)$. Depending on the antigen processing path- way, proteins produced through mammalian expression vectors can stimulate humoral or cellular immunity. Intracellular expression of a non-secreted antigen should specifically induce cellular immunity through presentation by the major histocompatibility complex class I (MHC I) molecules to cytotoxic $\mathrm{T}$ cells. During the immune response, many lymphokines are produced by the various cells involved, including IFN- $\gamma$, that can act

\section{Key words}

- DNA vaccine

- Gene therapy

Regulation

- Transcription 
on the target cell carrying the transgene. Modulation of expression of the transfected gene could occur and, depending on the promoter used, increase or decrease transgene expression. Therefore, the proper choice of promoter/enhancer linked to the gene of interest is critical to the success of DNA vaccinations.

We used the mammalian MHC I promoter/enhancer, BL3-6prmtr (7), and compared transgene expression and effects of IFN- $\gamma$ regulation to the popular viral promoter/enhancers of SV40 and CMV in various cell lines of several species. To date, almost all commercial mammalian expression vectors use viral promoter/enhancer sequences from pathogenic viruses such as simian virus 40 (SV40), or human cytomegalovirus (CMV). Although these promoter elements are from pathogenic viruses, they have become very useful due to high transcription initiation ability in most mammalian tissues (8). However, in genetic immunization applications involving human patients or animal husbandry, a mammalian promoter may be more desirable for easing

Table 1 - Cell lines used for transfections.

All cell lines were provided by the American Type Culture Collection (ATCC).

\begin{tabular}{lll}
\hline Species & Cell line & Tissue \\
\hline Mouse & A20.J & B lymphoma \\
& $\mathrm{C}_{2} \mathrm{C}_{12}$ & Myoblast \\
& G8 & Myoblast \\
& P815 & Mastocytoma \\
& RAW 264.7 & Macrophage \\
Human & COLO 320HSR & Colon carcinoma \\
& LCL 721.221 & B lymphoblast \\
& SW 837 & Rectal carcinoma \\
Cow & BL3.1 & B sarcoma \\
& MDBK & Kidney \\
Dog & D17 & Osteocarcinoma \\
Monkey & Vero & Kidney \\
Rat & NMU & Mammary carcinoma \\
Sheep & FLK & Kidney
\end{tabular}

public concern over transfecting DNA elements from pathogenic or tumor-causing viruses. BL3-6prmtr is the promoter for the cattle major histocompatibility complex class I gene. MHC I is expressed on nearly all tissues, is critical in immune system communication, and can be up-regulated by lymphokines such as IFN- $\gamma(9)$.

The SV40, CMV, and MHC I promoter/ enhancers have complex cis elements that bind varied cellular trans-factors (10-12). SV40 and MHC I promoters both have AP2 binding sites while CMV and MHC I promoters both have ATF binding sites. In common to all three promoters are AP1 and NFkB binding sites. Additionally, all three promoters have interferon response sequences. However, for CMV and SV40, factors binding to this region can inhibit transcription $(13,14)$, whereas, for MHC I, interferon response factors enhance transcription $(15,16)$.

\section{Comparison of luciferase expression driven by MHC I, SV40, and CMV promoter/enhancers}

The luciferase gene driven by BL3-6prmtr (cattle MHC I), SV40, or CMV promoter/ enhancer was used as a reporter gene transiently transfected in cell lines of various tissues from several species (listed in Table 1). Assays of luciferase production were used to compare the relative strengths of the MHC I promoter/enhancer to the viral promoter/enhancers transgene expression abilities in each cell line. Each of the three promoters was able to drive luciferase expression in all cell lines tested. However, BL36 prmtr was, in many cases, weaker than either SV40 or CMV promoters. The data, summarized in Table 2, show that SV40 was stronger than BL3-6prmtr in 12/14 cell lines and CMV was stronger in 6/14 cell lines. Comparisons between SV40 and CMV promoter/enhancers could not be made because the vector constructs were not similar. 


\section{Effect of IFN- $\gamma$ addition to transient transfected cell culture on transgene promoter}

Luciferase production was positively or negatively altered by IFN- $\gamma$ treatment depending on the promoter and cell line. Transiently transfected cell cultures were treated or not treated with rIFN- $\gamma$ for $16 \mathrm{~h}$ and then assayed for luciferase production. Results demonstrate that BL3-6prmtr-driven luciferase production increased in all cell lines, whereas the SV40 or CMV promoter-driven luciferase production decreased in most cell lines (Table 2).

Using the mammalian expression vectors p6/Luc (MHC I promoter driven) and pcDNA3/Luc (CMV promoter driven), we established stable transfects of several cell lines to ascertain whether IFN- $\gamma$ would affect chromosome integrated promoter/transgene expression differently than episomal transgene expression. We isolated neomycin-resistant clones of A20.J, P815, RAW 264.7, COLO 320HSR, SW 837, and BL3.1 cell lines and selected the highest luciferaseexpressing clones of each transgene construct. IFN- $\gamma$ treatment of these stable transfects produced similar results (Table 2) using the same IFN- $\gamma$ dosage $(500 \mathrm{U} / \mathrm{ml})$ and measurement time point $(16 \mathrm{~h}$; data not shown).

Many gene delivery vaccination applications use muscle as the target tissue (17-19). Our results indicate that while SV40 and CMV promoters drive luciferase expression in myoblasts to higher levels than BL36 prmtr, addition of IFN- $\gamma$ drastically reduces SV40- and CMV promoter-driven expression while increasing BL3-6prmtr-driven expression. Figure 1 gives a graphic illustration of this change.

\section{Kinetics of IFN- $\gamma$-treated stable transfect luciferase production}

IFN- $\gamma$ dosage and kinetics of luciferase
Table 2 - Effect of IFN- $\gamma$ on luciferase transgene production driven by $\mathrm{MHC} \mathrm{I}$, SV40, or CMV promoters.

Note: Increased expression after IFN- $\gamma$ treatment is in bold numbering. Decreased expression after IFN- $\gamma$ treatment is in italics numbering. aThree transfections per cell line of each luciferase gene construct (in parentheses) driven by M HC I, SV40, or CMV promoters were done for each experiment. Transfections were performed using the cationic lipid method (LipofectAMINE; Life Technologies, Inc., Gaithersbourg, MD). At least three experiments were done for each cell line. Transfection efficiency was measured using internal controls for either ß-galactosidase (pGL6 and pGL2 Control) or CAT (p6/Luc and pcDNA3/Luc). bIFN- $\gamma$ was added (+) or not added (-) to transient transfects which were assayed for luciferase production $16 \mathrm{~h}$ later. Human riFN- $\gamma$ was added to human, cow, dog, monkey, and sheep cell lines while mouse rIFN- $\gamma$ was added to mouse and rat cell lines (see Table 1). Numbers represent mean picogram (pg) luciferase per transfection calculated using a luciferase standard curve. The coefficient of variation was less than $10 \%$ for each experiment.

\begin{tabular}{|c|c|c|c|c|c|c|c|c|}
\hline \multirow[t]{3}{*}{ Cell line } & \multicolumn{4}{|c|}{$\mathrm{MHCI}$} & \multirow{2}{*}{\multicolumn{2}{|c|}{$\frac{\text { SV40 }}{\text { (pGL2 Control) }}$}} & \multirow{2}{*}{\multicolumn{2}{|c|}{$\frac{\text { CMV }}{(p c D N A 3 / L u c)}$}} \\
\hline & \multicolumn{2}{|c|}{$(p G L 6)^{a}$} & \multicolumn{2}{|c|}{ (p6/Luc) } & & & & \\
\hline & $(-)^{b}$ & $(+)$ & $(-)$ & $(+)$ & $(-)$ & $(+)$ & $(-)$ & $(+)$ \\
\hline A20.J & 30 & 41 & 50 & 81 & 826 & 409 & 126 & 16 \\
\hline BL3.1 & 40 & 54 & 7 & 10 & 8 & 5 & 4 & 2 \\
\hline $\mathrm{C}_{2} \mathrm{C}_{12}$ & 19 & 52 & 20 & 61 & 403 & 83 & 182 & 87 \\
\hline COLO & 56 & 107 & 30 & 53 & 28 & 11 & 11 & 2 \\
\hline D17 & 38 & 40 & 14 & 16 & 88 & 68 & 30 & 22 \\
\hline FLK & 36 & 251 & 99 & 234 & 215 & 354 & 59 & 89 \\
\hline G8 & 28 & 96 & 9 & 17 & 810 & 209 & 53 & 23 \\
\hline 721.221 & 34 & 39 & 19 & 20 & 43 & 18 & 18 & 17 \\
\hline MDBK & 8 & 9 & 8 & 10 & 10 & 9 & 13 & 8 \\
\hline NMU & 24 & 63 & 91 & 176 & 250 & 75 & 154 & 34 \\
\hline P815 & 28 & 30 & 4 & 5 & 57 & 34 & 4 & 2 \\
\hline RAW & 20 & 74 & 12 & 25 & 50 & 176 & 9 & 11 \\
\hline SW837 & 8 & 9 & 9 & 10 & 16 & 12 & 8 & 7 \\
\hline Vero & 34 & 62 & 14 & 35 & 208 & 150 & 10 & 7 \\
\hline
\end{tabular}

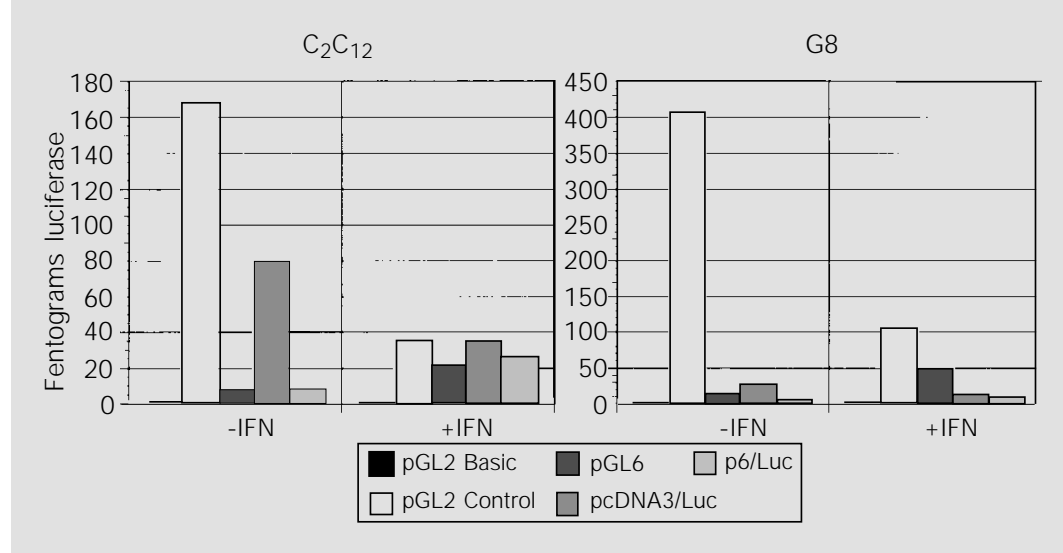

Figure 1 - BL3-6prmtr, SV40, and CMV promoter-driven luciferase production in transfected myoblasts. The mouse myoblast cell lines, $\mathrm{C}_{2} \mathrm{C}_{12}$ and $\mathrm{G} 8$, were transfected with luciferase gene constructs driven by no promoter (pGL2 Basic), the SV40 promoter/enhancer (pGL2 Control), the CMV promoter/enhancer (pcDNA3/Luc), or the cattle MHC class I promoter/ enhancer (pGL6; p6/Luc). Transfectant cell cultures were either untreated (-IFN) or treated (+IFN) with IFN- $\gamma$ for $16 \mathrm{~h}$ and then assayed for luciferase production. Three experiments of three transfections of each luciferase construct were performed. Measurements represent the mean relative light units (RLU) with coefficient of variation of less than $10 \%$. 
Figure 2 - Kinetics of luciferase transgene expression in stably transfected cell lines. Human colon carcinoma (COLO 320HSR) or mouse macrophage (RAW 264.7) cell clones with a chromosome-integrated luciferase transgene driven by either the CMV promoter (CMV/LuC) or the MHC I promoter (MHC I/LuC) were cultured in 24-well plates and treated with IFN- $\gamma(500 \mathrm{U} /$ $\mathrm{ml}$ ) for $0,4,8,24,48$, and $72 \mathrm{~h}$. Cells were harvested in $100 \mu \mathrm{l}$ lysis buffer at these time points and lysate was stored at $-70^{\circ} \mathrm{C}$ until assayed for luciferase. Graphs show the mean relative light units (RLU) and standard deviations of four experiments. expression driven by CMV or MHC I promoters in these stable transfects were measured. These data showed an optimal IFN- $\gamma$ dosage of 100-500 U/ml for both CMV and MHC I promoters for highest luciferase production at all time points (data not shown). The kinetics of luciferase transgene production showed that the amount of CMV-driven luciferase product peaked at approximately $8 \mathrm{~h}$ and then dropped to below baseline (untreated) levels at 16-24 h, while the MHC I-driven luciferase product continued to rise through $48 \mathrm{~h}$. Figure 2 shows these results using stably transfected human colon carcinoma (COLO 320HSR) and mouse macrophage (RAW 264.7) cell line clones.

\section{Effect of mixed lymphokine addition to transfected cell culture on transgene expression}

An in vivo immune response that was either initiated or promoted by the transgene would release a multitude of lymphokines, along with IFN- $\gamma$, that may have additive,

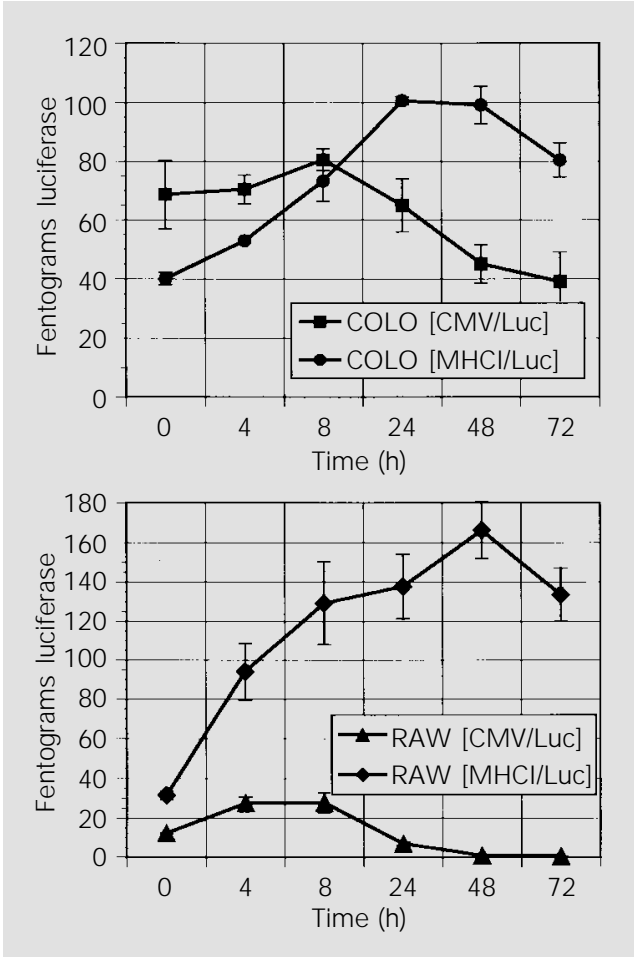

synergistic, or antagonistic effects on the transgene promoter. For example, tumor necrosis factor-alpha (TNF- $\alpha$ ), another lymphokine produced during an immune response, has been shown to inhibit CMV transcription but acts synergistically with IFN- $\gamma$ in enhancing MHC I transcription $(20,21)$. Using the supernatant from mitogen-stimulated $\mathrm{T}$ cells as a source of immune response lymphokines, we tested transgene expression in response to these mixed lymphokines as well as IFN- $\gamma$ in a transiently transfected human colorectal carcinoma cell line. The results, shown in Figure 3, indicate that the $\mathrm{T}$ cell blast supernatant increased transgene expression driven by MHC I promoter to an even greater extent than IFN- $\gamma$ alone. Viral promoter-driven expression was up-regulated by the mixed lymphokines compared to IFN- $\gamma$-treated cells but not to the level of untreated cells.

\section{Discussion}

Foremost in DNA vaccine delivery vector design should be the proper choice of promoter/enhancer to drive expression of the transferred gene. This choice is dependent upon target cell type as well as the functional goal of the transgene. DNA vaccine applications are designed to affect immune function to protect against a pathogenic agent, or lymphokine gene transfer to fight cancer. These applications stimulate the immune system resulting in production of many lymphokines that can directly act on the transfected target cell and thus indirectly act on the transgene promoter. At least one of these lymphokines, IFN- $\gamma$, is a potent antiviral agent, and has been shown to inhibit viral transcription of SV40 and CMV $(13,14)$, although many promoters for transgene expression are from SV40 or CMV $(2,17-19,22-$ 25). Our objective was to determine what effect IFN- $\gamma$ had on transgene expression driven by SV40, CMV, or MHC I promoter/ enhancers using cell lines of various tissues 
to ascertain the best choice for gene delivery vector promoter in immunoregulatory applications.

Our results show a down-regulation effect of IFN- $\gamma$ on both SV40 and CMV promoter/enhancer-driven transgene in most cell lines tested and an up-regulation effect of IFN- $\gamma$ on the MHC I promoter/enhancerdriven transgene in all cell lines tested. We note, however, that even with IFN- $\gamma$ downregulation, viral promoter-driven expression was frequently greater than that of IFN- $\gamma$ upregulated MHC I promoter-driven expression. Nonetheless, these negative selective pressures may shorten the expressible life of the transgene. For example, we noticed in our stably transfected cell clones that luciferase expression driven by BL3-6prmtr stayed at the same level for many cell passages whereas luciferase expression driven by CMV promoter showed a "dampening" of expression soon after cloning. This theory has yet to be tested.

In fact, studies using the stable transfects showed an ever increasing amount of luciferase from MHC I promoter-driven transgene over 48-72 h while amounts of lu- ciferase from transfect cultures of CMV promoter-driven transgene were near or below baseline levels at this time. These experiments showed an initial increase in CMV promoter-driven production peaking at around $8 \mathrm{~h}$ and then dropping to below baseline levels. Also, after 48 h, MHC I promoter-driven transgene product levels began to drop. We believe that these results were due to the cells being plated at $70 \%$ confluence at the beginning of the experiment, and to the fact that the medium was not changed through the 72-h period. Therefore, the slight increase in CMV promoter-driven luciferase production may reflect increased cell numbers to confluence rather than an IFN- $\gamma$-induced enhancement. The IFN- $\gamma$ inhibitory effect on the CMV promoter and enhancing effect on the MHC I promoter probably occurred within $4 \mathrm{~h}$. The reason MHC I promoter-driven luciferase production began dropping by 48-72 h may have been due to lack of fresh medium (growth factors) in the culture and perhaps the beginning of cell death. Since luciferase is a fairly stable protein, these data indicate that as long as IFN- $\gamma$ is present, the MHC I promoter

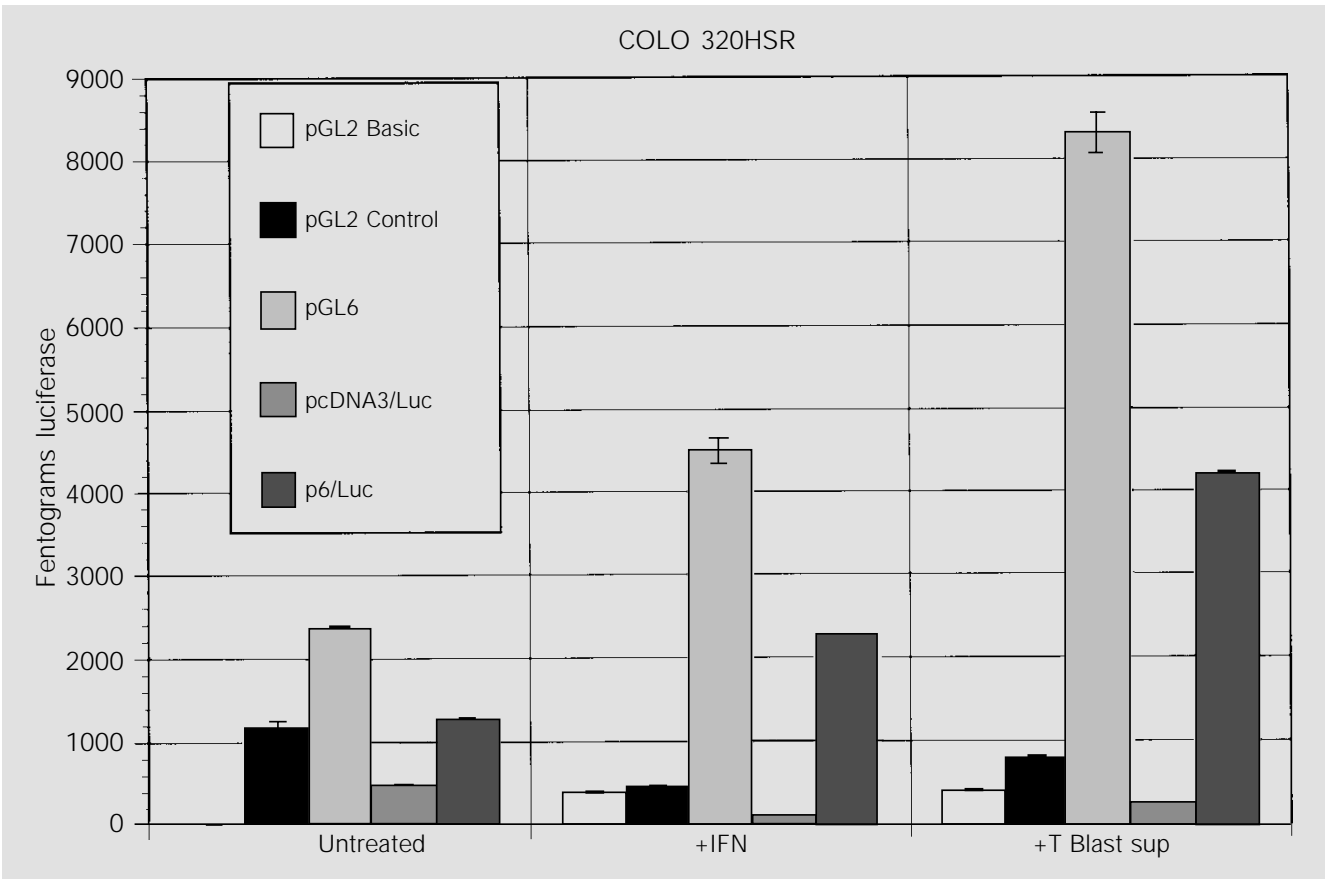

Figure 3 - Effect of IFN- $\gamma$ and T cell blast supernatant (sup) on BL3-6prmtr, SV40, and CMV promoter-driven luciferase production in transfected human colorectal carcinoma cells. The human colorectal carcinoma cell line COLO 320HSR was transfected with luciferase gene constructs driven by no promoter (pGL2 Basic), the SV40 promoter/enhancer (pGL2 Control), the HCMV promoter/enhancer (pcDNA3/Luc), or the cattle MHC class I promoter/enhancer (pGL6; p6/Luc). Transfected cell cultures were untreated or treated with either IFN- $\gamma$ (+IFN) or $10 \%$ supernatant from $3 \mathrm{~d} \mathrm{mi-}$ togen-stimulated $\mathrm{T}$ cells $(+\mathrm{T}$ Blast sup) and then assayed for luciferase production. Three experiments of three transfections of each luciferase construct were performed. Measurements represent the mean relative light units (RLU) with a coefficient of variation of less than $10 \%$. 
is up-regulated and the CMV promoter is down-regulated.

Although the type of cell or tissue is important in determining whether IFN- $\gamma$ as well as other immune response lymphokines will enhance or inhibit transgene expression driven by the viral promoters, our results indicate that most probably expression will be down-regulated. The MHC I gene, on the other hand, is a critical immune system communication molecule and has been shown to have enhanced transcription due to lymphokines produced during an immune response (9). The use of $T$ cell blast supernatanttreated transfected cell lines confirmed the enhanced expression of BL3-6prmtr-driven transgenes, similar to the results shown in Figure 3, for all cell lines tested, including COLO 320HSR, LCL 721.221, BL3.1, and FLK (data not shown).

The MHC I promoter/enhancer was used to drive luciferase in two different promoters and, as seen in Table 2, caused different luciferase expressions. Vectors pGL2 Control (SV40 driven) and pGL6 (MHC I driven) were standard reporter gene vectors typical for use in transient transfection assays. Vectors pcDNA3/Luc (CMV driven) and p6/ Luc (MHC I driven) were engineered from a mammalian expression vector containing a separate, selectable neomycin gene independently driven by the SV40 promoter/enhancer. This vector is typical for use in stable transfections. Even though the same MHC I promoter/enhancer drove expression of the same luciferase gene, the lower expression results of p6/Luc compared to pGL6 could be due to transcription factor competition by the SV40 promoter or some inhibitory effect by the neomycin gene product, as others have shown $(26,27)$. The fact that many selectable marker genes in mammalian gene expression vectors are under viral promoter control should be another consideration in choosing the best gene delivery vector for immune response applications. Whether the transgene was integrated in the genome or episomal did not alter the general effect of IFN- $\gamma$ on a particular cell line according to our stable or transient transfection results using pcDNA3/ Luc and p6/Luc.

We still need to elucidate whether these data represent effects of an actual immune response to the transgene typical in DNA vaccination. Intramuscular DNA vaccination of mice with rabies virus glycoprotein gene driven by SV40 or MHC I promoter developed a comparable antibody response (28). However, co-inoculation of mouse IFN$\gamma$ plasmid and either of the SV40- or MHC Idriven rabies vaccine vectors resulted in a reduced antibody and $\mathrm{T}$ helper cell proliferation response in these mice. Cattle vaccinated with bovine herpesvirus-1 glycoprotein $\mathrm{D}(\mathrm{gD})$ gene driven by CMV or MHC I promoter delivered by the biolistic process resulted in a greater $\mathrm{gD}$-specific antibody response to the $\mathrm{CMV} / \mathrm{gD}$ vaccine compared to the MHC $\mathrm{I} / \mathrm{gD}$ vaccine (data not shown). In both of these examples the measurement of the DNA vaccine response was based on antibody titer or $\mathrm{T}$ helper cell proliferation immune responses that depend upon MHC II antigen presentation. Therefore, the protein produced by the transgene had to be secreted by the transfected cell, taken up and processed by antigen presenting cells, and presented in the context of MHC II to B and T helper cells. To truly assess the efficacy of the MHC I versus the viral DNA vaccine promoters in vivo, studies must be done on the long-term or memory response. Our data suggest that, although MHC I is a weaker promoter than the viral promoters, immune response cytokines could eventually shut down the viral promoter while augmenting the MHC I promoter. Thus there may be long term protection with DNA vaccines driven by the MHC I promoter. Additionally, studies must be done on cytotoxic $\mathrm{T}$ cell responses of MHC I versus viral promoterdriven vaccines. Because the cytotoxic $T$ cell response involves antigen presentation by MHC I (most likely in the transfected 
cell), the lower but longer term expression of MHC I promoter may induce a superior cytotoxic $\mathrm{T}$ cell response compared to viral promoter-driven vaccines.

We believe the MHC I promoter/enhancer, BL3-6prmtr, would be a better choice for gene delivery vector promoter in immunoregulatory applications than the widely used viral promoters of SV40 and CMV. This belief is based on the generally inhibitory effect of IFN- $\gamma$, as well as other lymphokines, on SV40 and CMV promoterdriven transgene expression, and the amplified effect of IFN- $\gamma$ and other lymphokines on MHC I-driven transgene expression. Many researchers using viral promoters to express transgenes have been frustrated by the short- lived expression of these transgenes. Our results offer an explanation for these observations. Admittedly, the viral promoters of SV40 and CMV are generally stronger than the MHC I promoter, BL3-6prmtr. Nevertheless, our data suggest that for long-term sustained transgene expression necessary for an optimal immune response, the mammalian MHC I promoter/enhancer could be a preferable option.

\section{Acknowledgments}

We thank the Instituto de Ciências Biológicas, UFMG, especially in the person of Dr. Vasco Azevedo, for his effort in making this conference possible.

\section{References}

1. Ertl HCJ \& Xiang ZQ (1996). Genetic immunization. Viral Immunology, 9: 1-9.

2. Felgner PL \& Rhodes G (1991). Gene therapeutics. Nature, 349: 351-352.

3. Gilboa E \& Smith C (1994). Gene therapy for infectious diseases: The AIDS model. Trends in Genetics, 10: 139-144.

4. Tighe $\mathrm{H}$, Corr $\mathrm{M}$, Roman $\mathrm{M} \&$ Raz $E$ (1998). Gene vaccination: plasmid DNA is more than just a blueprint. Immunology Today, 19: 89-97.

5. Thompson L (1992). At age 2, gene therapy enters a growth phase. Science, 258: 744-746.

6. Xiang ZQ, Pasquini $\mathrm{S}$, He Z, Deng $\mathrm{H}$, Wang Y, Blaszczyk-Thurin MA \& Ertl HCJ (1997). Genetic vaccines - a revolution in vaccinology? Springer Seminars in Immunopathology, 19: 257-268.

7. Harms J S, Li W \& Splitter GA (1995). The cattle major histocompatibility complex (MHC) class-I possesses HLA-like promoters. Gene, 160: 249-252.

8. Cheng L, Ziegelhoffer PR \& Yang NS (1993). In vivo promoter activity and transgene expression in mammalian somatic tissues evaluated by using particle bombardment. Proceedings of the National Academy of Sciences, USA, 90: 44554459.

9. Singer DS \& Maguire JE (1990). Regulation of the expression of class I MHC genes. Critical Reviews in Immunology, 10: 235-256.
10. Espel E, Fromental $C$, Reichenbach $P \&$ Nabholz M (1990). Activity and interleukin 1 responsiveness of SV40 enhancer motifs in a rodent immature T cell line. EMBO J ournal, 9: 929-937.

11. Ghazal P, Lubon H, Reynolds-Kohler C, Hennighausen L \& Nelson J A (1990). Interactions between cellular regulatory proteins and $a$ unique sequence region in the human cytomegalovirus major immediateearly promoter. Virology, 174: 18-25.

12. Israel A, Le Bail O, Hatat D, Piette J, Kieran $M$, Logeat $F$, Wallach $D$, Fellous $M$ \& Kourilsky P (1989). TNF stimulates expression of mouse MHC class I genes by inducing an NFkB-like enhancer binding activity which displaces constitutive factors. EMBO J ournal, 8: 3793-3800.

13. Gribaudo G, Ravaglia S, Caliendo A, Cavallo R, Gariglio M, Martinotti MG \& Landolfo S (1993). Interferons inhibit onset of murine cytomegalovirus immediate-early gene transcription. Virology, 197: 303-311.

14. Kerr IM \& Stark GR (1992). The antiviral effects of the interferons and their inhibition. J ournal of Interferon Research, 12: 237-240.

15. Korber B, Mermod N, Hood L \& Stroynowski I (1988). Regulation of gene expression by interferons: Control of $\mathrm{H}-2$ promoter responses. Science, 239: 13021306.

16. Shirayoshi Y, Burke PA, Appella E \& Ozato
K (1988). Interferon-induced transcription of a major histocompatibility class I gene accompanies binding of inducible nuclear factors to the interferon consensus sequence. Proceedings of the National Academy of Sciences, USA, 85: 5884-5888.

17. J iao $S$, Williams $P$, Berg RK, Hodgeman BA, Liu L, Repetto G \& Wolff J A (1992). Direct gene transfer into nonhuman primate myofibers in vivo. Human Gene Therapy, 3: 21-33.

18. Ulmer J B, Donnelly JJ, Deck RR, Dewitt $C M$, Dwarki VJ , Felgner PL, Freidman A, Hawe LA, Leander KK, Martinez D, Parker SE, Perry HC, Rhodes GH, Shiver JW, Montgomery DI \& Liu MA (1993). Heterologous protective immunity to influenza A by intramuscular injection of DNA encoding a conserved viral protein. Science, 259: 1745-1749.

19. Yang NS (1992). Gene transfer into mammalian somatic cells in vivo. Critical Reviews in Biotechnology, 12: 335-356.

20. Blanchet $\mathrm{O}$, Bourge J F, Zinszner H, Tatari Z, Degos L \& Paul P (1991). DNA binding of regulatory factors interacting with MHC-class-I gene enhancer correlates with MHC-class-I transcriptional level in class-I-defective cell lines. International J ournal of Cancer, Suppl 6: 138-145.

21. Pavic I, Polic B, Cmkovic I, Lucin P, J onjic S \& Koszinowski UH (1993). Participation of endogenous tumour necrosis factor $\alpha$ in host resistance to cytomegalovirus in- 
fection. J oumal of General Virology, 74: 2215-2223.

22. Manthorpe $M$, Cornefert-J ensen $F$, Hartikka J , Felgner J, Rundell A, Margalith M \& Dwarki V (1993). Gene therapy by intramuscular injection of plasmid DNA: Studies on firefly luciferase gene expression in mice. Human Gene Therapy, 4: 419-431.

23. Schmidt EV, Christoph G, Zeller R \& Leder P (1990). The cytomegalovirus enhancer: A pan-active control element in transgenic mice. Molecular and Cellular Biology, 10: 4406-4411.
24. Takebe $Y$, Seiki M, Fujisawa J, How P, Yokota K, Arai K, Yoshida M \& Arai N (1988). SR alpha promoter: an efficient and versatile mammalian cDNA expression system composed of the simian virus 40 early promoter and the R-U5 segment of human T-cell leukemia virus type 1 long terminal repeat. Molecular and Cellular Biology, 8: 466-472.

25. Thompson TA, Gould MN, Burkholder J K \& Yang NS (1993). Transient promoter activity in primary rat mammary epithelial cells evaluated using particle bombardment gene transfer. In Vitro Cellular and
Developmental Biology, 29A: 165-170.

26. Artelt $P$, Grannemann $R$, Stocking $C$, Friel J, Bartsch J \& Hauser H (1991). The prokaryotic neomycin-resistance-encoding gene acts as a transcriptional silencer in eukaryotic cells. Gene, 99: 249-254.

27. Valera A, Perales J C, Hatzouglou M \& Bosch $F$ (1994). Expression of the neomycin-resistance (neo) gene induces alterations in gene expression and metabolism. Human Gene Therapy, 5: 449-456.

28. Xiang ZQ, He Z, Wang Y \& Ertl HCJ (1997). The effect of interferon- $\gamma$ on genetic immunization. Vaccine, 15: 896-898. 